\title{
A High-Throughput, Multiplex Cell Death Assay Using an RNAi Screening Approach
}

\author{
Katrina J. Falkenberg, 1,2 Darren N. Saunders, 4,5 and Kaylene J. Simpson 1,2,3,6 \\ ${ }^{1}$ Victorian Centre for Functional Genomics, Peter MacCallum Cancer Centre, East Melbourne, Victoria 3002, \\ Australia; ${ }^{2}$ Department of Pathology, The University of Melbourne, Parkville, Victoria 3052, Australia; ${ }^{3}$ Sir Peter \\ MacCallum Department of Oncology, The University of Melbourne, Parkville, Victoria 3052, Australia; ${ }^{4}$ Cancer \\ Research Program, The Kinghorn Cancer Centre, Garvan Institute of Medical Research, Darlinghurst, New South \\ Wales 2010, Australia; ${ }^{5}$ St. Vincent's Clinical School, University of New South Wales Medicine, Sydney, New \\ South Wales 2000, Australia
}

This protocol outlines a high-throughput, multiplex cell death assay and its use in conjunction with a genome-scale siRNA screen to identify genes that cooperate with a drug to induce apoptosis. The assay, ApoLive-Glo (Promega), measures viability of drug-treated, reverse-transfected cells via the fluorescent CellTiter-Fluor reagent, which includes a substrate that is cleaved by a live cell protease. ApoLive-Glo also quantitates cell death by the amount of cleaved caspases 3 and 7 using a luminescent Caspase-Glo 3/7 caspase activation assay. The advantage of the multiplex assay is that it distinguishes rapid cell death from the slower activation of caspase activity, permitting measurement of different stages of cell death in the same sample at a single time point. In parallel, a high-content imaging protocol involving 4',6-diamidino-2-phenylindole-stained nuclei is used as a cost-effective way to quantitate viability of vehicle-treated control cells. Automation and robotic liquid handling are built into the protocol to increase speed of workflow and improve reproducibility. A screen using these assays will identify gene targets that are essential for viability irrespective of drug treatment and gene targets that cause a synergistic enhancement of cell death in the presence of drug. Candidate target activity can then be validated by conventional flow cytometry-based assays.

It is essential that you consult the appropriate Material Safety Data Sheets and your institution's Environmental Health and Safety Office for proper handling of equipment and hazardous materials used in this protocol.

\section{Reagents}

ApoLive-Glo multiplexed assay (includes Caspase-Glo 3/7 substrate, Caspase-Glo 3/7 buffer, GF-AFC substrate, and assay buffer; Promega G6411)

For consistency in a long-term screening project spanning weeks or months, it is important to prepare fresh Caspase-Glo 3/7 reagent every time, so order the appropriate pack size. The combined Caspase-Glo 3/7 substrate and buffer can be stored at $-20^{\circ} \mathrm{C}$ for later use, but storage decreases signal intensity by $\sim 25 \%$, so it should be used in independent experiments and not combined with fresh reagent. When calculating reagent quantities for a screen as well as preparing reagents for use, always include an extra $10 \%$ to account

\footnotetext{
${ }^{6}$ Correspondence: kaylene.simpson@petermac.org

(C) 2014 Cold Spring Harbor Laboratory Press

Cite this protocol as Cold Spring Harb Protoc; doi:10.1101/pdb.prot080267
} 
K.J. Falkenberg et al.

for pipetting error. This is especially important when working with large numbers of plates, where a small volumetric error can become large across many plates. When using liquid-handling automation, you must also prepare a dead volume. This is the extra volume required for priming (filling all the tubes), avoiding bubbles in the dispenser tubing, and accounting for reagent remaining in the reservoirs. Calculate how many plates you can process in one batch. The more plates you can handle at once, the less reagent is lost as dead volume (and the shorter the total screening time), although the additional 10\% volume needed for pipetting error is unaffected.

\section{Cells}

The ApoLive-Glo assay is most amenable to adherent cells. Suspension cells are very difficult to work with in high throughput format, particularly from the perspective of performing media changes with automation. In addition, suspension cells cannot be transfected with siRNAs. However, the ApoLive-Glo assay can be used with suspension cells in high throughput if no media change is required (e.g., compound screens). However, to be cost effective, cells must be plated in a minimum volume, which is feasible with adherent cells but not as feasible for suspension cells. Fixing and staining with DAPI is not feasible for suspension cell lines in high throughput. In low throughput, we recommend a cytospin.

CellTiter-Fluor (CTF) Cell Viability Assay (includes GF-AFC substrate and assay buffer;

\section{Promega G6082)}

This reagent is needed to supplement that which is supplied with the ApoLive-Glo assay, because of the dilution of the caspase component. GF-AFC substrate and buffer can be freeze-thawed as separate reagents. The combined substrate and buffer, called CTF reagent, can be stored at $4^{\circ} \mathrm{C}$ for up to 1 wk with minimal loss of activity but should not be frozen. For consistency, we prepare fresh CTF reagent immediately before use.

DAPI solution $(2 \times)$

To prepare $1 \mathrm{~mL}$ of $2 \times$ DAPI solution, combine $2 \mu \mathrm{L}$ of $5 \mathrm{mg} / \mathrm{mL} \mathrm{4}$,6-diamidino-2-phenylindole (DAPI) with $40 \mu \mathrm{L}$ of $10 \%$ Triton $X-100$ and $958 \mu \mathrm{L}$ of $50 \mathrm{mM}$ Tris ( $\mathrm{pH}$ 7.5). Minimize freeze/thaw cycles of DAPI.

Microplates with RNAi library, 384-well, opaque-walled, clear-bottomed (see Step 1)

Use white-walled plates for ApoLive-Glo and black-walled plates for DAPI staining. The choice of brand is determined by the optical abilities of the preferred imager and cost. If you are not performing high end, very detailed microscopy, a lower quality plate can be used, but be aware that use of such plates often correlates with problems such as uneven plate surface, which can impact imaging. All plates should be evaluated on a per instrument basis.

Paraformaldehyde (PFA) (4\% in Tris)

To prepare a $4 \%$ working solution of PFA, dilute one part of, 16\%, PFA (e.g., Electron Microscopy Sciences 15710) with three parts of Tris (50 $\mathrm{mm}, \mathrm{pH} 7.5)$.

PBS

Reverse transfection reagents

Tris (50 mM, pH 7.5), filtered

To make $1 \mathrm{~L}$ of this reagent, dissolve $6.057 \mathrm{~g}$ of Tris base in $800 \mathrm{~mL}$ of deionized $\mathrm{H}_{2} \mathrm{O}$. Adjust the solution to $\mathrm{pH}$ 7.5 with concentrated $\mathrm{HCl}$ and bring the volume to $1 \mathrm{~L}$ with deionized $\mathrm{H}_{2} \mathrm{O}$. Immediately before use, filter through a $0.45-\mu \mathrm{m}$ filter.

\section{Equipment}

The high-end instrumentation required for this protocol is generally installed in a core facility that is managed by automation specialists. These specialists, who are well versed in many assays, develop robust protocols for instrument usage and train users. They also assist with assay development and optimization as required.

Automation for liquid handling, capable of dispensing 5-50 $\mu \mathrm{L}$ (e.g., EL406 microplate washer dispenser; BioTek)

Centrifuge, with microplate buckets

Foil, aluminum (optional)

Freezer, preset to $-20^{\circ} \mathrm{C}$

Incubator, humidified, preset to $37^{\circ} \mathrm{C}$

Microplate barcode labeler (alphanumeric) and readers for high-throughput-enabled instrumentation (optional)

Barcode instruments allow the user to track plates and avoid errors caused by stacking plates in the wrong order or orientation. 
Microplate heat sealer, automated (optional)

This highly recommended item saves the user from sealing plates by hand. The seals are cut by the instrument to fit entirely within plate boundaries, preventing the plate-stacking instrumentation "gripper fingers" from becoming stuck on overhanging edges. If you instead choose to manually adhere seals, pay particular attention to overhangs.

Microplate reader, multiwell, capable of reading luminescence, with filters for CTF assay (excitation: 380-400 nm, emission: $505 \mathrm{~nm}$ ) and a dichroic mirror (e.g., Synergy H4 Hybrid Multi-Mode

Microplate Reader; BioTek)

Microplate seals, foil

Microplate stacker (optional)

Stackers automatically deliver microplates to the reader and microscope. Their even timing improves screening accuracy.

Microscope, high-throughput (i.e., high-content imager)

Orbital plate shaker, small, with variable speed option

Refrigerator, preset to $4^{\circ} \mathrm{C}$

Tissue culture equipment, including a biosafety cabinet

Uncouple the light from the operation of the cabinet so you have independent control of it (for the purposes of fixing and staining) when the unit is running. Few biosafety cabinets operate this way out of the box, but they are relatively easy to modify.

Water bath, preset to $37^{\circ} \mathrm{C}$

\section{METHOD}

The high-throughput screen described here consists of two parallel arms: One for cells treated with drug and one for cells treated with drug-free vehicle. An RNAi screen for identifying synthetic lethal targets is depicted in Figure 1 A. The drugtreated cells are evaluated for general viability using CTF and for apoptosis using Caspase-Glo 3/7. Together, these assays constitute the ApoLive-Glo multiplex assay. (For more information, see the ApoLive-Glo Multiplex Assay Technical Manual from Promega at http://www.promega.com/resources/protocols/technical-manuals/101/apolive-glo-multiplex-assay-protocol/.) The control, vehicle-treated cells are subjected to nuclear staining by DAPI followed by highcontent cell counting as a surrogate readout of cell death (it is a true readout of the combination of cell death and proliferation). Figure $1 B$ depicts the steps required to perform these assays and the time required for each step.

\section{RNAi Screen: Reverse Transfection and Treatment}

1. Reverse-transfect the cells with the siRNA library and lipid-based transfection reagent in 384-well, opaque-walled microplates according to the chosen transfection protocol.

i. Prepare duplicate plates for each treatment (control and drug-treated).

ii. Use clear-bottomed plates. For multiplexed CTF and Caspase-Glo 3/7 assays, use whitewalled plates; for CTF alone, use either black- or white-walled plates; and for fluorescence microscopy (e.g., DAPI), use black-walled plates.

Although white-bottom plates are often recommended for luminescence assays to eliminate cross-well luminescence bleed-through, the opaque bottoms prevent visualizing the cells, which is important for evaluating cell density, efficiency of positive controls, and general health of the cells, as well as for identifying contamination. We have found that the bleed-through of a high-responding well to an adjacent nonresponding well is minimal ( $1 \%-2 \%$ of the high-luminescence value).

iii. Plate cells in column 1 for mock transfection (lipid only) to evaluate the cell dispensing automation (during analysis, determine variance in that column to confirm that all rows of the plate were consistently dispensed).

iv. Include positive controls, both technical (e.g., PLK1 for cell death) and assay-specific (see Introduction: High-Throughput Approaches to Measuring Cell Death [Saunders et al. 2014]). We use columns 2 and 23 for our positive and negative controls.

See Troubleshooting. 
K.J. Falkenberg et al.

A

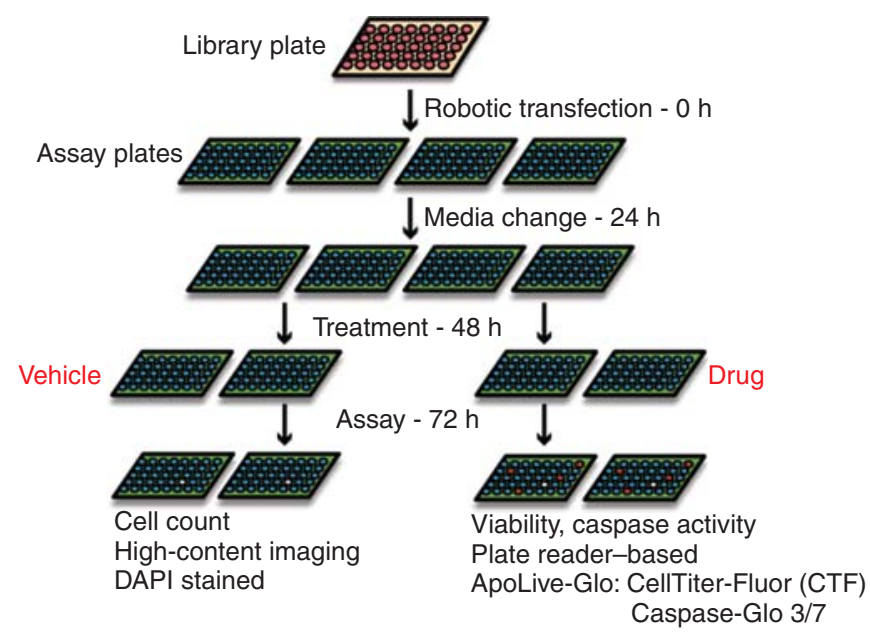

B

$$
\text { Drug }
$$

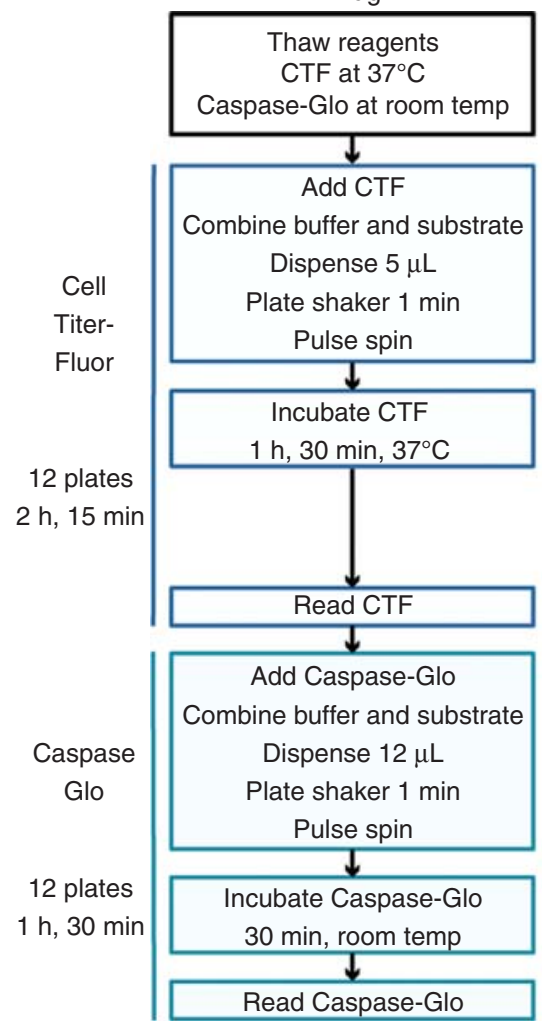

Vehicle

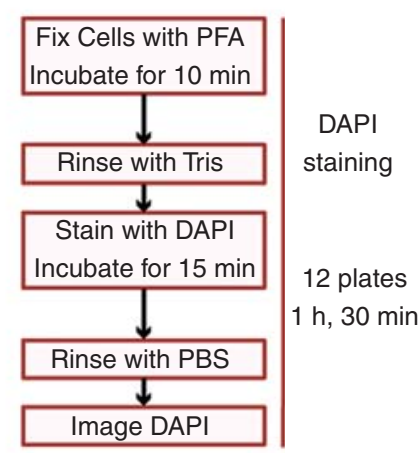

Total time excluding imaging $\sim 4 \mathrm{~h}$

FIGURE 1. Experimental workflow. (A) The experimental steps of a genome-scale siRNA screen. A single 384-well siRNA library plate was used to reverse transfect four assay plates. Final siRNA concentration was $40 \mathrm{~nm}$, and each well received lipid and cells. Plates were incubated for $24 \mathrm{~h}$ before changing the medium and a further $24 \mathrm{~h}$ before drug treatment. Duplicate plates received drug or the vehicle control. After $24 \mathrm{~h}$ of treatment, the vehicle plates were assayed for cell count using high-content imaging with DAPI nuclear staining. Drug-treated plates were assayed for viability and caspase activity using the plate reader-based ApoLive-Glo assay. $(B)$ The experimental steps of the ApoLive-Glo assay coupled with DAPI nuclear staining for quantitation of cell number on the vehicle control. The time to complete each segment of the assay is described. To decrease overall hands-on time, DAPI staining is performed in parallel with the ApoLive-Glo incubations and quantitation.

v. Include a nontargeting siRNA as a negative control.

vi. Leave one column free of cells as a "medium only" control. This important control is used for subtracting a background reading for plate reader-based assays. We use column 24 of the 384-well plate for this purpose, but whichever column you choose, stay consistent once the 
choice made. If you choose to work in 96-well plates there is far less room for controls; therefore, we suggest to create an additional "sentinel plate" that is medium alone, treated the same as the target plates and averaged to provide a medium-only background value.

vii. Consider the total number of plates and workflow. We are able to process six siRNA library plates in one transfection session of $20 \mathrm{~min}$, resulting in 24 experimental assay plates (two for the vehicle arm and two for the drug-treated arm for each library plate).

2. Incubate plates with drug or vehicle control. Each well should contain $20 \mu \mathrm{L}$.

\section{The Assays}

All assay steps are conducted at room temperature unless otherwise stated. When using multiple plates, always run the plates in the same order for each experimental step and for each assay when multiplexing. This ensures that the workflow timing remains consistent and provides a fail-safe if the barcode reader is unable to detect a barcode. Once work has begun on a set of plates, it must be completed to the end of the procedure.

3. Thaw the GF-AFC substrate and assay buffer at $37^{\circ} \mathrm{C}($ a $10-\mathrm{mL}$ bottle of buffer takes $\sim 10 \mathrm{~min}$ in a water bath).

4. Thaw the Caspase-Glo 3/7 lyophilized substrate and buffer at room temperature while the CTF assay is being performed (be prepared; a 10-mL bottle of buffer takes $\sim 2 \mathrm{~h}$ to thaw on the bench!).

Alternatively, the Caspase-Glo $3 / 7$ reagents can be thawed overnight at $4{ }^{\circ} \mathrm{C}$, but remember to equilibrate them to room temperature before use.

\section{CTF Assay}

CTF is a viability assay that makes use of a fluorogenic, cell-permeable peptide substrate. Live cells take up the substrate, and constitutive protease activity cleaves it to the fluorescent form, which generates a fluorescent signal proportional to the number of live cells. This protease activity is only retained in live cells with intact plasma membranes. When membrane integrity is lost, the protease is inactive and the fluorescent signal reduced. The reduction in fluorescent signal corresponds to the reduction in numbers of live cells.

5. Prepare the CTF reagent by combining $10 \mu \mathrm{L}$ of GF-AFC substrate with $2.5 \mathrm{~mL}$ of assay buffer. Mix well.

6. With an automated dispenser, add $5 \mu \mathrm{L}$ of CTF reagent to each well of the plates of cells that have been treated with drug.

The volume added in this step is CRITICAL, because it is the amount that must be added to the $20 \mu L$ of medium already in each well of the 384-well plate.

7. Shake the plates on an orbital plate shaker for 1 min at $\sim 1 / 4$ of maximum speed.

8. Centrifuge the plates briefly (pulse-spin them) to $60 \mathrm{~g}$ at room temperature to remove reagents from the sides of the wells.

9. Incubate the plates at $37^{\circ} \mathrm{C}$ in a humidified incubator for $1.5 \mathrm{~h}$.

To accelerate the workflow, the control plates that were not treated with drug can be fixed and DAPI-stained during the CTF incubation (Steps 17-28) (see Fig. 1B).

10. Read the plates with a plate reader: measure the fluorescence at $380-400 \mathrm{~nm}_{\mathrm{Ex}} / 505 \mathrm{~nm}_{\mathrm{Em}}$. Settings for each instrument must be determined empirically, and once determined, must remain constant throughout the screen. Increase the gain, sensitivity, or integration time (luminescence) to increase signal intensity and dynamic range (the difference between the positive and negative controls).

While the CTF plates are being assayed on the plate reader, high-throughput imaging of the DAPI-stained plates can be initiated (Step 29).

See Troubleshooting.

\section{Caspase-Glo 3/7 Assay}

Caspase-Glo 3/7 is a luminescent end point assay measuring the combined activity of caspases 3 and 7, the executioner caspases and central mediators of the intrinsic and extrinsic apoptosis pathways. The assay contains a lumino- 
K.J. Falkenberg et al.

genic caspase 3/7 substrate, containing the tetrapeptide sequence DEVD. Addition of the combined Caspase-Glo 3/7 reagent results in immediate lysis of cells, allowing cleavage of the caspase substrate into its mature form, which is a luciferase substrate. The luciferase reaction releases light, the amount of which is proportional to the amount of caspase activation in the well.

11. Add the thawed bottle of Caspase-Glo 3/7 buffer from Step 4 to the substrate and mix well. Keep the mix protected from light by using it in a biosafety cabinet with the light off.

12. With an automated dispenser, dispense $12 \mu \mathrm{L}$ of substrate mix to each well of the 384 -well plates that were taken through the CTF assay.

This addition creates a 1:2 reagent dilution with the $25 \mu \mathrm{L}$ of medium already in each well. Refreeze any remaining reagent at $-20^{\circ} \mathrm{C}$.

13. Shake the plates on an orbital plate shaker for $1 \mathrm{~min}$ at $\sim 1 / 4$ of maximum speed.

14. Centrifuge the plates briefly (pulse-spin them) to $60 \mathrm{~g}$ at room temperature to remove reagents from the sides of the wells.

15. Incubate the plates at room temperature for exactly $30 \mathrm{~min}$.

16. Measure luminescence with a plate reader.

See Troubleshooting.

\section{Nuclear Staining for Cell Counting}

Nuclear staining should be performed on the plates of cells that were not treated with drug (i.e., the vehicle-treated cells). Evaluate all liquid-handling steps to ensure that cell attachment to the plate is not disturbed during the fixing and staining steps.

17. Aspirate the contents of each well using a high aspirate setting. We leave $\sim 15 \mu \mathrm{L} /$ well, but this volume depends on the adherence capacity of the cells being used.

18. Add a volume of $4 \%$ PFA in Tris that is equal to the volume left behind after aspiration (in our case, $15 \mu \mathrm{L}$ ), for a final concentration of $2 \%$ PFA.

For good coverage in a 384-well plate, aim for $\sim 25 \mu \mathrm{L}$ final volume. You can optimize for a lower volume if you want to conserve reagents. Alternatively, if you have good, adherent cells and a low volume $(\sim 5 \mu \mathrm{L})$ remaining after aspirating, you can dilute the PFA to $2 \%$ in advance and use that directly.

19. Incubate the plates for $10 \mathrm{~min}$ at room temperature.

20. Aspirate the contents of each well.

21. Rinse each well with $50 \mu \mathrm{L}$ of $50 \mathrm{~mm}$ Tris ( $\mathrm{pH} 7.5$ ).

This volume removes most of the PFA, which can interfere with the clarity of DAPI images.

22. Aspirate the contents of each well using a high aspirate setting; leave $\sim 10 \mu \mathrm{L} /$ well.

23. Add $10 \mu \mathrm{L}$ of $2 \times$ DAPI solution, resulting in a final concentration of $5 \mu \mathrm{g} / \mathrm{mL}$ DAPI.

As in Step 18, if the volume remaining after aspiration is low, a $1 \times$ DAPI solution can be added.

The $2 \times$ DAPI solution contains Triton X-100 to permeabilize cells in conjunction with staining, effectively combining these steps.

24. Incubate the plates for 15 min with the lights off in a biosafety hood.

Alternatively, to reduce the exposure to light, cover the top plate in the microplate stack with foil or another plate.

25. Aspirate the contents of each well.

26. Add $50 \mu \mathrm{L}$ of PBS to each well.

This volume prevents evaporation leading to dried wells and allows subsequent restaining with other fluorophores.

27. Centrifuge the plates briefly (pulse-spin them) to $60 \mathrm{~g}$ at room temperature to remove reagents from the sides of the wells.

28. Seal the plates with foil seals, ideally by using an automated heat sealer. 
Data Analysis

29. Image the plates on a high-content imager immediately, or store the plates at $4^{\circ} \mathrm{C}$ wrapped in foil before imaging.

DAPI is very robust, so the plates can be stored at least a week before imaging, but this timing depends on which other fluorophores are used.

See Troubleshooting.

30. Store the plates wrapped in foil at $4^{\circ} \mathrm{C}$ in case they need to be reimaged.

The methods below describe how to analyze the CTF, Caspase-Glo 3/7, and cell counting data to generate a list of screen hits (see Fig. 2). Briefly, the cell counting data from DAPI nuclear staining is analyzed using cell number and field count values to reveal the wells that are dead in the vehicle arm. These wells can be removed from downstream analysis in the drug arm. Next, CTF and Caspase 3/7 data from the drug-treated arm undergo normalization, followed by identification of the most-reduced CTF wells (synthetic lethal cell death hits) and the most-increased Caspase-Glo 3/7 wells (sensitizer hits).

\section{Quantitation of Cell Number}

Perform cell counting on the plates of cells that were treated with vehicle and then stained with DAPI.

31. Average the replicate plates for cell count and field number.

32. To evaluate the health of the well, define a binning strategy for cell counts. If the predetermined cell count (based on control healthy wells) cannot be reached within the predetermined field number, the well conditions have a toxic effect. As long as the predetermined cell count is reached, field number gives an indication of the health of the well. In the example shown in Figure 3, we set a count of 1500 cells in up to 25 fields, but these numbers will vary among screens and depend on cell line, sensitivity to death stimulus, etc.:

- Toxic bin: $<1500$ cells counted in 25 fields

- Very healthy bin: $\geq 1500$ cells counted in $\leq 11$ fields (note: the negative controls consistently count $\geq 1500$ cells in $7-8$ fields)

- Moderately healthy bin: all other wells ( $\geq 1500$ cells counted in $12-25$ fields, inclusive) See Discussion.

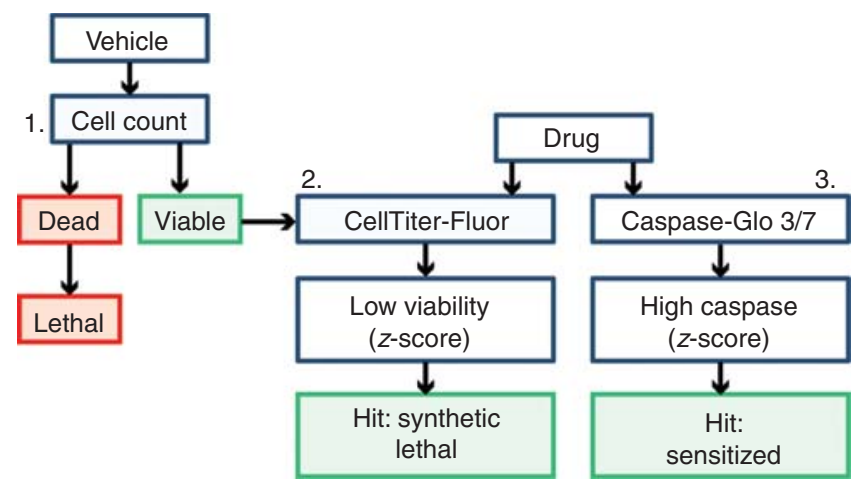

FIGURE 2. Analysis pipeline, combining screen arms (vehicle and drug) and assays (cell counting, CTF, and CaspaseGlo 3/7). (1) DAPI cell counting on the vehicle-treated plates determines viability on gene knockdown alone. In an siRNA screen, a well is considered dead, and the siRNA lethal, if the cell number threshold is not reached. These gene targets are excluded from further analysis in the drug-treated arm. A well is viable if the cell number threshold is reached. The combined effect of gene knockdown and drug treatment on viable wells is evaluated with (2) CTF and (3) Caspase-Glo 3/7. CTF hits are those wells with a low-viability z-score, where a synthetic lethal interaction has taken place between gene knockdown and drug treatment. Caspase-Glo 3/7 hits are those wells with a high-caspase activation $z$-score (see Fig. 4). These wells are sensitized to drug treatment, because they show substantial caspase activity but are not yet dead. The same target may fall into both the reduced viability and caspase activation hit bins because they are not mutually exclusive, but in practice we find this situation uncommon. In the first instance, following convention, $z$-score cutoffs of $z \leq-2$ or $z \geq 2$ are a starting point for defining hits, but cutoffs may be determined empirically as in the case of the screen described, based on the range and spread of the data and subsequent validation considerations (Birmingham et al. 2009; see also Fig. 4). 
K.J. Falkenberg et al.
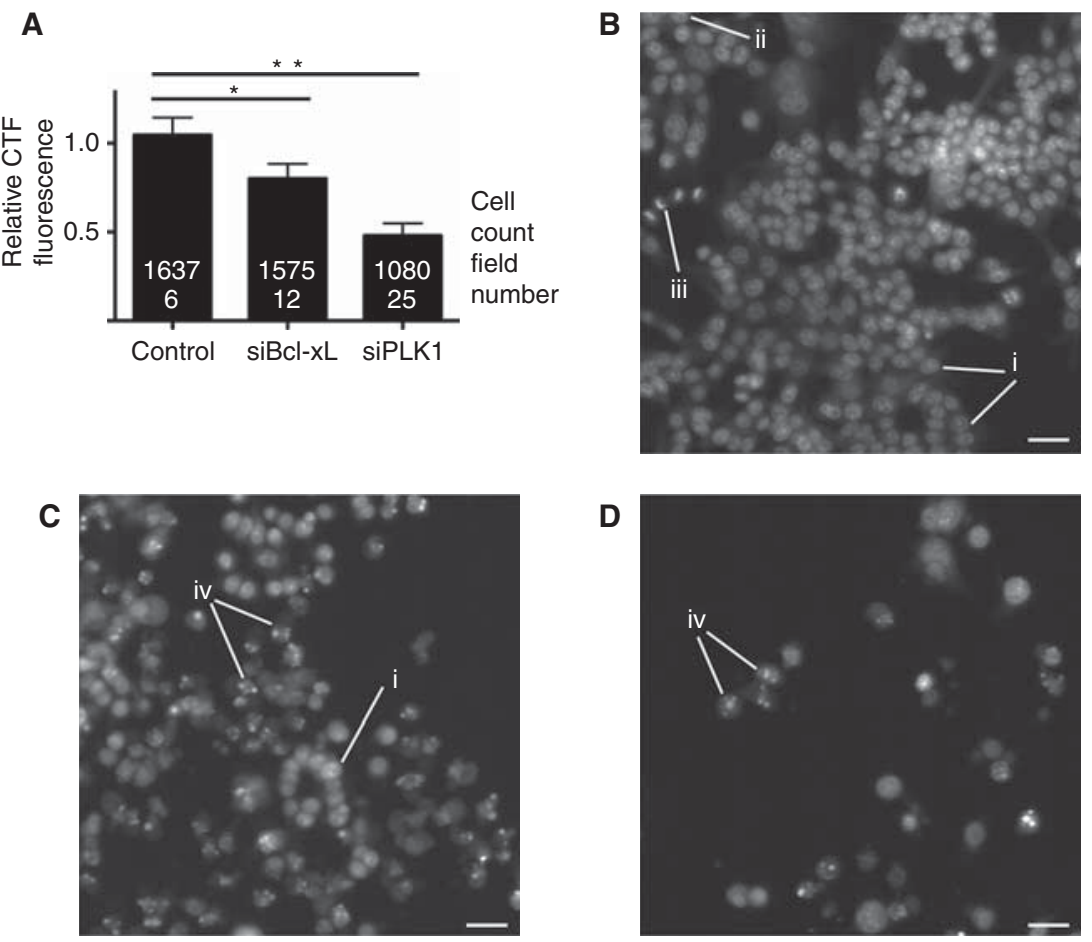

FIGURE 3. Concordance between CTF and DAPI cell counting. (A) Results for CTF and DAPI cell counting showing excellent correlation between the two assays. Cells underwent lipid-based reverse transfection with 40 nM siRNA in duplicate 384-well plates and were incubated for $72 \mathrm{~h}$ before being subjected to CTF or DAPI staining. PLK1 knockdown greatly reduces cell viability as measured by CTF. Bcl-xL knockdown reduces viability to a smaller extent. Data are expressed relative to lipid transfection (mock), mean, and standard deviation from a single experiment plotted. Lipid transfection gives a very healthy well, counting 1500 cells in only six fields. Bcl-xL knockdown reduces viability such that it takes six more fields to achieve this cell number (total of 12 fields). PLK1 knockdown is toxic to the cells. Even in 25 fields, we are unable to count 1500 cells. ${ }^{*} p \leq 0.01,{ }^{* *} p \leq 0.001$. Representative high-content images of DAPI-stained cells $(B)$ mock, $(C)$ siBcl-xL, and $(D)$ siPLK1 showing different levels of cell death within the wells. Specific features are illustrated: (i) healthy nuclei, (ii) an object falling on the edge of the field which is thereby excluded from the analysis, (iii) a cell undergoing division, and (iv) apoptotic nuclei included in the cell count. Scale bar, $30 \mu \mathrm{m}$.

33. Evaluate the readouts for each control type on every plate to ensure that they all reach the threshold value within the same field counts. The coefficient of variation (CV) measurement can be used here: For a given number of replicates of the same treatment, the CV, expressed as a percentage, is the standard deviation divided by the average (see Introduction: High-Throughput Approaches to Measuring Cell Death [Saunders et al. 2014]). If the variability between cell number and field count for each type of control is $>25 \%$, the assay is too variable to statistically interpret any hits.

34. If the screen is performed over many weeks, compare the raw data on a "per plate" and "per week" basis to ensure that the cells are performing as expected. A fold change ratio of positive to negative controls can also be compared weekly to ensure consistency.

\section{CTF and Caspase-Glo 3/7 Analysis}

Perform these analyses on the plates of drug-treated cells.

35. Average the luminescence signal from the media-only column, and subtract this background from all other values.

36. Similar to the analysis of the DAPI-stained cells, ensure that the positive and negative controls on each plate have reported raw and normalized values that are consistent with optimization and 
data on a weekly basis. Calculate the CV (in \%) to ensure that variability is within an acceptable range.

37. Calculate the $Z^{\prime}$-factor for each positive control relative to the same technical negative control (e.g., the PLK1 death control versus the nontargeting siRNA negative control). The $Z^{\prime}$-factor is a measure of the dynamic range between the positive and negative control and should, at the very least, be $>0$. If a plate fails, it must be rescreened (see Introduction: High-Throughput Approaches to Measuring Cell Death [Saunders et al. 2014]).

38. Perform one of the following analysis options.

- Perform sample-based normalization: In an unbiased screen, a $z$-score (or robust $z$-score) can be calculated for all samples (Birmingham et al. 2009). The $z$-score is a measure of the number of standard deviations from the mean (or, in the case of robust $z$-scores, absolute deviations from the median). This normalization is appropriate only when most of the samples are assumed to be negative.

- Perform control-based normalization: An alternative analysis is to generate fold change to a negative control. This should be done on a per plate basis and can be used for biased data sets. Either this method or the $z$-score method can be performed when working with a limited number of plates.

39. When analyzing data from an entire RNAi screen, it is necessary to reduce any potential bias from plate to plate or week to week, in addition to factoring in the layout of the library (e.g., if an entire genome is plated in gene families, a kinome plate may yield many more hits than a plate of uncharacterized targets from the remaining genome). The sample data from each library plate is normalized to the average of the technical negative control samples on each plate, and then the replicate plates are averaged. The averaged value for each individual siRNA target is then collated and the robust $z$-score is calculated across the library collection (use the same approach for a genome or small custom library).

40. Define a cutoff for $z$-score or fold change based on positive and negative controls. A $z$-score cutoff of $z \geq 2$ or $z \leq-2$ is commonly used (Birmingham et al. 2009). The cutoff can be made more stringent for screens with a large dynamic range and depending on the number of hits desired for further analysis. Our laboratory routinely takes the top 400-500 candidates through for secondary analysis, therefore a decision is made on the actual $z$-score cutoff for selection based on the number of candidates that reflect this. In the example shown in Figure 4A, there is a skewed distribution that might be expected from a screen set up to identify death. If we were to take all the Caspase-Glo targets from a $z$-score cutoff of 2 , there would have been several thousand targets, an unmanageable amount. By defining the number of targets to pursue in a validation screen, you can then specify the $z$-score cutoff for caspase activation and for cell viability. For comparison, Figure 4B shows an example of a more normal, less-skewed data set by which targets that decrease and increase viability can be identified from either end of the $z$-score distribution.

\section{TROUBLESHOOTING}

Problem: Cells detach from the plate during media changes.

Solution: Automated liquid handlers can subject cells to more powerful forces during aspiration and dispensing than hand pipetting does. Keep in mind that when hand pipetting, we place the tip down the side of the well to avoid disturbing cells, whereas most liquid handling robots aspirate and dispense with vertical tips. Minimize the number of washing steps where possible, and test for $z$ heights (vertical distance above the plate) that allow cells to remain adhered to the plate. Optimize liquid aspiration steps to remove as much liquid as possible without disturbing cell adhesion (the volume remaining must be factored in with all subsequent calculations for volumes required for each assay). Plating density is also crucial; if cells are too sparse, they are more likely to come off. The rate of liquid dispensing is a compromise between high accuracy 
K.J. Falkenberg et al.
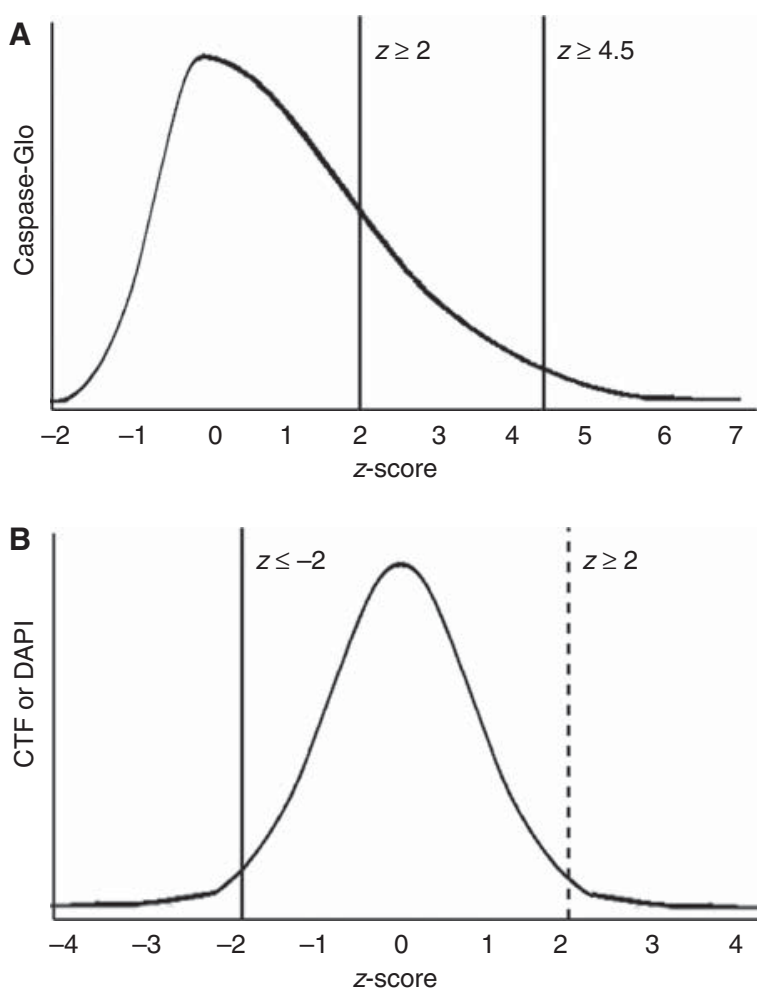

FIGURE 4. A z-score analysis of CTF and Caspase-Glo 3/7 assays. Representative cartoon plots illustrate the types of $z$-score distribution curves and subsequent cutoff values that could be achieved for a genomescale screen for $(A)$ Caspase-Glo 3/7 and $(B)$ CTF or DAPI. The schematic in $(A)$ shows a skewed distribution of response to caspase-mediated cell death (Caspase-Glo 3/7) where a long tail has thousands of targets with a $z$-score cutoff $>2$. Moving to a $z$-score cutoff of 4.5 clearly changes the number of targets that will be studied. The schematic in $B$ shows a more normal data distribution for cell viability based on CTF or counting of DAPI-stained nuclei, where there are targets outside $>2$ and $<-2$ that increase and decrease viability, respectively. In interpreting these figures, the values on the $y$-axis are not important; emphasis is placed on the profile of the distribution of the $z$-score across the entire data set (the $x$-axis).

(fast flow rate) and low mechanical force to the cells (low flow rate). We dispense at a high flow rate to improve accuracy, but to reduce the impact of media dispensing on cells, we installed an angled deflector shield on the dispense head of the Biotek 406 dispenser (our liquid handler of choice). The angled shield reduces the downward force by shifting the dispensed media to offcenter.

If cell adhesion issues persist, a cellular substrate such as poly-L-lysine or an extracellular matrix such as collagen or fibronectin can be used. These substrates do not interfere with the Caspase-Glo 3/7 and CTF assays, but their presence can lead to optical issues during microscopy, such as changes in the focal plane across the well. Before using them, evaluate substrates for your specific cell line and assay. We do not recommend firmly adhering cells if you are investigating the cytoskeleton and migration.

Problem (Step 1): Assay-specific positive controls cannot be identified.

Solution: To accurately determine the dynamic range of your assay and the potential of the screen to identify hits, you need an assay-specific positive control. This should be the same entity that you are screening: If you are conducting an siRNA screen, you need an siRNA, and for a compound screen, you need a drug. Although a drug can be used in assay development, it will not reflect any variability that might arise with weekly transfections. If a positive control for your biological system is unknown (which is common), an in-depth literature search can help. If you cannot identify a positive control, you can still complete a screen, but be aware that gain settings, for example, might need to be altered to accommodate higher readings than calibrated when the screen was developed. If all else fails, technical controls such as known genes or drugs that cause cell death can be used to generate at least some expectation of the extent of data values.

Problem: A plate's barcode is not being read.

Solution: Apply the following procedures to ensure that barcodes are always read.

- Make sure that the barcodes are correctly placed on the plate (the barcode should be level and placed in the middle of the side of the plate). 
- Avoid scratching the barcode with a fingernail or other apparatus (gloves or not, this is a common problem when applying barcodes).

- If there is a mirror involved in the barcode reader, clean it weekly with $70 \%$ ethanol to prevent dust from causing barcode reader errors.

Problem (Steps 10 and 16): There is too much noise in the assay, the background is too high, or the dynamic range of the plate reader-based assay is too low.

Solution: Improving your assay's signal-to-noise ratio increases dynamic range and reduces noise in the assay. As much as possible, reduce background. For the plate reader-based assays, keep control wells healthy by allowing the cells sufficient nutrients (make sure to change the medium during the assay) and space (do not grow the cells to overconfluence). Reagents do not always need to be used at the manufacturer's specified concentration or dilution, so you can experiment with amounts and concentrations to get cleaner, more robust data.

Problem (Steps 10 and 16): The assay's dynamic range changes over time.

Solution: The luminescent readout of the Caspase-Glo 3/7 assay is very sensitive to changes in incubation time, so the incubations for all plates must be equal. You might have to scale down the number of plates done in one batch if, for example, the time it takes to read them means the plates at the end are sitting around for longer.

Problem (Steps 10 and 16): Fluorescence or luminescence readings are saturated or out of range.

Solution: Maxed-out readings can happen when you have optimized the instrument gain based on control wells that respond to a lesser degree than some of the screen hits. To avoid maxing out with very high readings, take care not to set the gain too high. Plates with saturated signals can be read again with a lower gain. If this modification is not applied to all of the plates, plate-based normalization must be used.

Problem (Step 29): High-content imaging shows high background or low clarity.

Solution: Residual PFA remaining in the wells after fixation can make images fuzzy, so it is important to remove as much of it as possible. In addition, the three-dimensional nature of matrices compared to the plastic they are coating can make it difficult to find a focal plane. Try to reduce the amount of matrix/substrate coating the wells, or use a higher $z$ height to avoid using any substrate at all. Optimize for a high signal-to-noise ratio, especially when using fluorophore-conjugated antibodies.

\section{DISCUSSION}

\section{High-Content Microscopy}

Each instrument has its own proprietary tools for software-driven data analysis, but cell counting is very standard. During optimization, pay close attention to defining the average nucleus size for the population, particularly for excluding dead and dying cells. In the example shown in Figure 3, working in 384-well format and at a magnification of $20 \times$ on a Cellomics ArrayScan Vti microscope, we imaged 1500 cells or 25 fields, whichever came first. This field number was chosen, because it includes the entire center of the well but excludes fields on the edge of the well, where cell confluence was often different than in the center. The cutoff of 1500 cells was chosen by observing control healthy wells in comparison with moderately healthy and dead wells under a light microscope followed by nuclear staining, counting, and statistical evaluation. Control, healthy wells reached 1500 cells in few fields (seven to eight fields), and moderately healthy wells reached this cutoff with fields to spare (15 to 20 fields), but clearly apoptotic wells were unable to make this cell count threshold in the 25 fields (see Fig. 3). There is no hard-and-fast rule dictating the required number of cells to be imaged. Rather, the number of cells will be based on the phenotype of interest, 
K.J. Falkenberg et al.

the size of cells, the percentage of positive cells in control wells (penetrance of the phenotype), and robust statistical outcomes. As a general guideline, it is usually sufficient to image between 800 and 2000 cells, and we recommend this as a ballpark figure when getting started. Cells bordering fields were excluded from the analysis to prevent cells being counted multiple times. Note that for simple cell counting, it is also possible to use lower magnification to significantly reduce the amount of data generated and the processing time required.

\section{Selecting, Configuring, and Validating the Multiplex Assay}

We have presented here a strategy for performing a high-throughput siRNA screen with a multiplexed cell death assay readout to identify genes that when knocked down cooperate with a drug to induce apoptosis (i.e., synthetic lethality). For this screen, we required a plate reader-compatible, highthroughput assay that would specifically detect apoptosis, as opposed to total cell number. Assays based on ATP content and mitochondrial metabolism, such as CellTiter-Glo (Promega) and alamarBlue (Invitrogen), respectively, were unsuitable because they measure a combination of proliferation and death (Fig. 5A,B). Detection of caspase 3/7 activation, however, using either fluorescent- or luminescent-based detection (Promega), provided a robust readout that correlated with traditional flow cytometry-based annexin V staining (Fig. 5C,D). The luminescent assay had greater sensitivity and dynamic range than the fluorescent version of the assay. The end point nature of this assay (requiring lysis of cells) raised the possibility of false negatives arising from wells that had undergone very rapid apoptosis, resulting in a loss of cells and insignificant caspase activity at the assay end point. To counter this problem, we included a viability assay that would distinguish rapid cell death from slower development of caspase activity within the window of our assay. The advantage of this mul-

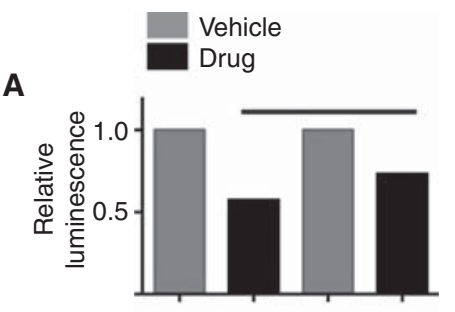

B

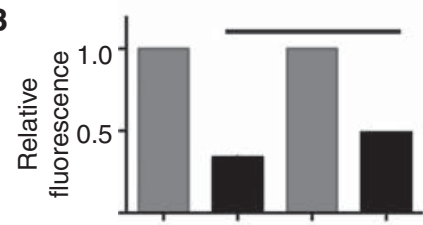

C

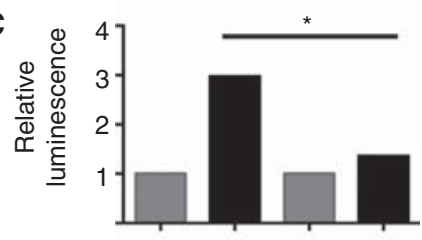

D

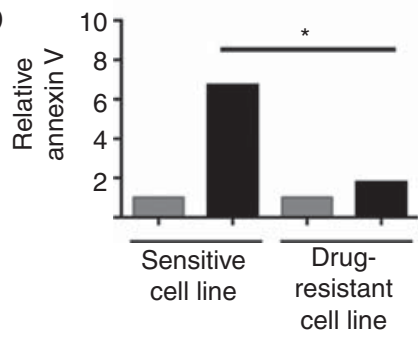

FIGURE 5. Finding an appropriate cell death readout. A schematic comparison modeled on experimental data of different cell viability readouts using cell lines that are sensitive or resistant to drug treatment. (A) CellTiter-Glo measures ATP content. (B) alamarBlue measures mitochondrial metabolism. Both assays show a significant difference between vehicle and drug treatment for both sensitive and drug-resistant cells, deeming them unsuitable for the screen. (C) Caspase-Glo 3/7 correlates significantly with $(D)$ annexin $\mathrm{V}$ staining, proving the suitability of the caspase reagent for high-throughput use. Cells were treated with drug or vehicle for $24 \mathrm{~h}$ before being subjected to the relevant assay. Data are expressed relative to the vehicle mean. Asterisk represents a theoretical statistical significance in cell death between the sensitive and resistant lines after drug treatment. 
tiplex format is that we could identify different stages of cell death in the same sample at a single time point.

In an attempt to reduce reagent cost, we evaluated the CTF and Caspase-Glo 3/7 reagents at 1:1 (recommended by the manufacturer), 1:2, and 1:4 dilutions. We found that signal intensity and dynamic range was maintained for Caspase-Glo 3/7 at a 1:2 dilution but was lost at a 1:4 dilution. No further dilution of CTF was possible without severely abrogating the assay signal. To further reduce assay cost, we chose not to use ApoLive-Glo for the vehicle control arm of the screen and instead used high-content imaging to count cell number after fixation and nuclear staining with DAPI. We imaged 1500 cells in up to 25 fields and determined the relative "health" of the well by the number of fields it took to sample 1500 cells (i.e., we used field number as a measure of viability). We showed that cell count correlated significantly with CTF-determined viability (Fig. 3). Intensity of DAPI fluorescence, as a measure of DNA content, is also a useful gross overall indicator of cell cycle distribution (e.g., $2 \mathrm{~N}$, $4 \mathrm{~N})$. This analysis allowed us to identify conditions causing strong cell cycle arrest in G1 or G2/M phase but was not sensitive enough to evaluate more subtle differences in cell cycle profile. If cell cycle analysis is central to your screen, we recommend including a dedicated cell cycle marker such as Ki67, phosphorylated histone H2B, or DNA synthesis via BrdU/EdU incorporation (Poon et al. 2008).

We show that three different gene targets that caused synthetic lethality as measured by caspase 3/7 activity were further validated by conventional low-throughput flow cytometry-based assays for phosphatidylserine externalization (annexin V binding), DNA fragmentation (subG1 DNA content with propidium iodide [PI]), and caspase activation (cleaved caspase 3 intracellular staining) (Fig. 6). These assays confirm the utility of the ApoLive-Glo assay for identifying apoptosis in a high-throughput, plate reader-based format.

A

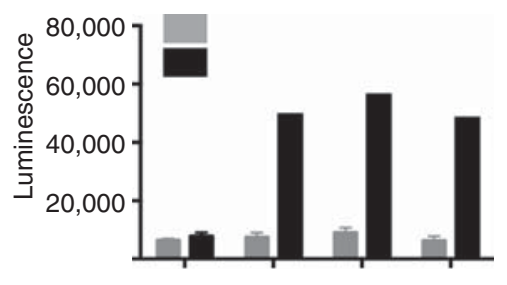

B

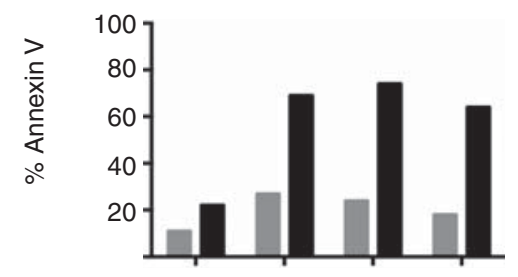

C

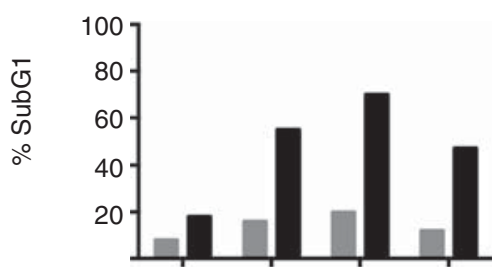

D

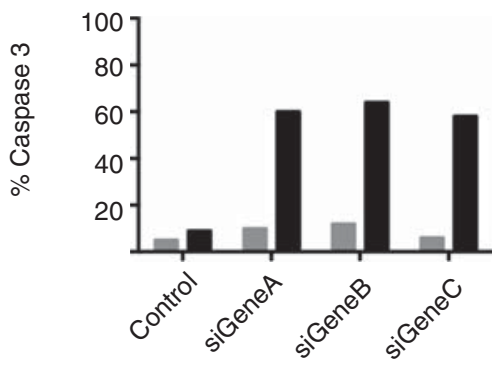

FIGURE 6. Confirmation of plate reader assays using standard FACSbased readouts. A schematic representation modeled on experimental data comparing three individual gene targets between the plate readerbased caspase activation assay and standard FACs readouts for cell death. Effect of gene knockdown with and without drug treatment using (A) Caspase-Glo 3/7 (raw luminescence units), (B) annexin V staining (percent cells), (C) subG1 DNA content (percent cells), and $(D)$ intracellular cleaved caspase 3 staining assays (percent cells). Results reproduce in all assays, confirming the utility of Caspase-Glo 3/7 for identifying apoptotic cells. 
We thank members of the Victorian Centre for Functional Genomics (VCFG), Kate Gould for bioinformatics analysis of screen data, and Daniel Thomas and Yanny Handoko for expert technical guidance on all automation and screening support. The assay development was funded by Merck Sharp and Dohme, and an Australian National Health and Medical Research Council (NHMRC) project grant \#1028871 (to Professor Ricky Johnstone, Peter MacCallum Cancer Centre). K.J.F. is a recipient of an Australian Postgraduate award. D.N.S. is supported by the NHMRC, New South Wales Office of Science and Medical Research, Cancer Institute New South Wales, and the Mostyn Family Foundation. The VCFG (KJS) is funded by the Australian Cancer Research Foundation (ACRF), the Victorian Department of Industry, Innovation and Regional Development (DIIRD), the Australian Phenomics Network (APN) supported by funding from the Australian Government's Education Investment Fund through the Super Science Initiative, the Australasian Genomics Technologies Association (AMATA), the Brockhoff Foundation and the Peter MacCallum Cancer Centre Foundation.

\section{REFERENCES}

Birmingham A, Selfors LM, Forster T, Wrobel D, Kennedy CJ, Shanks E, Santoyo-Lopez J, Dunican DJ, Long A, Kelleher D, et al. 2009. Statistical methods for analysis of high-throughput RNA interference screens. Nat Methods 6: 569-575.

Poon SS, Wong JT, Saunders DN, Ma QC, McKinney S, Fee J, Aparicio SA. 2008. Intensity calibration and automated cell cycle gating for high- throughput image-based siRNA screens of mammalian cells. Cytometry A 73: 904-917.

Saunders DN, Falkenberg KJ, Simpson KJ. 2014. High-throughput approaches to measuring cell death. Cold Spring Harb Protoc doi: 10.1101/pdb.top072561. 


\section{A High-Throughput, Multiplex Cell Death Assay Using an RNAi Screening Approach}

Katrina J. Falkenberg, Darren N. Saunders and Kaylene J. Simpson

Cold Spring Harb Protoc; doi: 10.1101/pdb.prot080267

\begin{tabular}{rc}
$\begin{array}{r}\text { Email Alerting } \\
\text { Service }\end{array}$ & Receive free email alerts when new articles cite this article - click here. \\
\hline $\begin{array}{r}\text { Subject } \\
\text { Categories }\end{array}$ & $\begin{array}{c}\text { Browse articles on similar topics from Cold Spring Harbor Protocols. } \\
\text { Apoptosis Assays (73 articles) } \\
\text { High-Throughput Analysis, general (155 articles) } \\
\text { RNA Interference (RNAi)/siRNA (123 articles) }\end{array}$ \\
& \\
\hline
\end{tabular}

